Networking Reception on Wednesday, April 24, 7:30-9:00 pm. This new event is for attendees who want to showcase their research in an interactive and fun way. More information is available on the 2019 MRS Spring Meeting website.

The MRS/Cambridge University Press Publications Booth 100 will be featured in the Exhibit Hall. Stop by to learn about the MRS Publications Portfolio and receive $20 \%$ off all book purchases.

The popular Science as Art competition is open to all registered Meeting attendees. Multiple first-place and secondplace awards of $\$ 400$ and $\$ 200$, respectively, will be presented at the Meeting. Guidelines are listed on the 2019 MRS Spring Meeting website.

The deadline to preregister for the 2019 MRS Spring Meeting is April 5 (5:00 pm ET). International travelers

\begin{tabular}{|l|c|c|}
\hline \multicolumn{2}{|c|}{2019 MRS SPRING MEETING REGISTRATION RATES } \\
\hline Meeting Registration & $\begin{array}{c}\text { PREREGISTRATION } \\
\text { before 5:00 pm (ET) } \\
\text { April 5, 2019 }\end{array}$ & $\begin{array}{c}\text { ON-SITE REGISTRATION } \\
\text { after 5:00 pm (ET) } \\
\text { April 5, 2019 }\end{array}$ \\
\hline Meeting Registration with MRS Member Discount & $\$ 715$ & $\$ 845$ \\
\hline Student Registration (Proof of student status required.) & $\$ 565$ & $\$ 705$ \\
\hline Student Registration with MRS Member Discount & $\$ 130$ & $\$ 200$ \\
\hline Retired & $\$ 190$ & $\$ 160$ \\
\hline Unemployed & $\$ 160$ & $\$ 215$ \\
\hline
\end{tabular}

2019 MRS Spring Meeting registrations include MRS Membership from July 1, 2019 to June 30, 2020.

are reminded to begin the visa process early. For additional information on the Spring Meeting, contact MRS Member Services, e-mail info@mrs.org and tel. 724-779-3003.
The MRS website can be accessed for updated information on confirmed talks and details on special events, information on obtaining a visa, and preregistration at.mrs.org/spring2019.

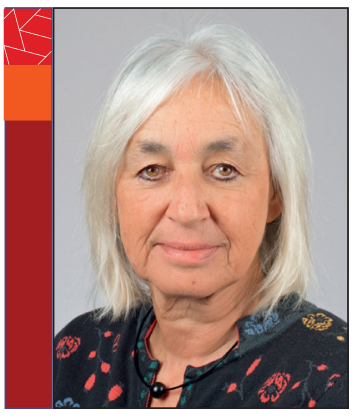

\section{Van Swygenhoven-Moens to present Kavli lecture during 2019 MRS Spring Meeting plenary session}

$\mathrm{H}$ elena Van Swygenhoven-Moens will present The Fred Kavli Distinguished Lectureship in Materials Science on Tuesday, April 23. She is a professor at the École Polytechnique Fédérale de Lausanne (EPFL) in the Materials Science Institute; she also leads the Neutrons and X-rays for Mechanics of Materials Laboratory and heads the Photons for Engineering and Manufacturing Research Group at the Paul Scherrer Institute in Switzerland.

Helena's presentation is titled, "Synchrotron Light to Investigate Materials In Operando." Synchrotron radiation beams are now sufficiently bright and detectors are sufficiently fast that scattering and x-ray absorption data can be followed in real time for a material under action. With the use of synchrotron radiation, high-resolution computer-aided tomographic microscopy is possible. One can now look at the formation of pores, microcracks, or gas bubbles during the interaction of a material with an external environment.

Helena studied physics at the Vrije Universiteit Brussels and received her $\mathrm{PhD}$ degree in physics from the Central Jury in Belgium. After a professional break for motherhood, she continued her career with a Marie-Heim Vögtlin Grant from the Swiss National Science Foundation. Helena is a Fellow of the Materials Research Society, elected member of the Royal Academy of Science of Belgium, and an advanced grant holder from the European Research Council. For many years, she chaired the International Board of the International Committee of Strength of Materials, was a member of the Scientific Advisory Committee of the European Spallation Source, was on the peer review panel of Diamond, and was on the beamline review panels of ESRF. Helena also serves on the engineering expert panel of the National Science Foundation Flanders and on expert panels of the ERC.

The core of her work is the development and use of in situ experiments at synchrotron and neutron facilities, with the aim to follow a material's microstructure in operando and to provide synergies between experiments and computational modeling. Helena's research focuses on the link between synthesis and microstructure, including laser-based additive manufacturing methods and the connection between microstructure and mechanical behavior of a variety of materials, ranging from nanostructured materials for watch components, superelastic alloys for medical applications, and advanced steels and lightweight alloys for structural applications. Through her ERC advanced grant (MULTIAX), Helena addresses non-proportional multiaxial straining covering the gap between our current knowledge of mechanical behavior derived from uniaxial deformation tests and the engineering reality of applications and processing routes. 
Phoenix Convention Center, North Building, 300 Level, Exhibition Halls C-E

Tuesday, April 23, 2:00 pm - 7:00 pm • Wednesday, April 24, 11:00 am - 7:00 pm

Admiral Instruments

Booth 832

www.admiralinstruments.com

Potentiostats/Galvanostats; EIS

Measurements; Photo-Electrochemistry

AdValue Technology, LLC Booth 603

www.advaluetech.com

Alumina, Quartz and Sapphire Products

\section{AIP Publishing}

Booth 804

publishing.aip.org

Physics Journals; Conference Proceedings;

Digital Archive

\section{AJA International, Inc.}

Booth $\mathbf{5 0 0}$

www.ajaint.com

Sputtering/E-Beam/lon Milling; Sputtering

Sources; Target and Evaporation Materials

\section{Allwin21 Corp.}

Booth 229

www.allwin21.com

Rapid Thermal Processing; Plasma

Asher Descum Etcher; Sputtering

Deposition System

\section{American Physical Society Booth 705}

journals.aps.org

Journals

\section{ANCORP}

Booth 404

www.ancorp.com

Vacuum Flanges and Fittings; Vacuum Valves; Vacuum Chambers

\section{Anton Paar}

Booth 700

www.anton-paar.com

Atomic Force Microscope; Gas Sorption;

Pycnometers

\section{Arizona State University}

Booth 430

www.asu.edu

Educate Graduates; Materials Research;

Innovation

\section{Barnett Technical Services \\ Booth 330 \\ www.barnett-technical.com \\ Micromanipulators; Cathodoluminescence; \\ AFM; Raman; Polariscopes}

Bio-Logic USA

Booth 326

www.bio-logic.net

Potentiostats; Impedance Analyzers;

Battery Cyclers
Blue Wave Semiconductors, Inc. Booth 400

www.bluewavesemi.com

Electron Beam Evaporators; Pulsed Laser

Deposition; Diamond CVD-HFCVD

Bruker Corporation

Booth 629

www.bruker.com

Analytical Equipment; SEM Detectors

Cambridge University Press | Booth 100

Materials Research Society

www.cambridge.org

Books; Journals

\section{Cell Press}

Booth 800

www.cell.com

Journals; Periodicals

\section{CrystalMaker Software Ltd.}

Booth 405

www.crystalmaker.com

CrystalMaker 10.4; CrystalDiffract 6;

SingleCrystal 3

Delcom Instruments, Inc.

Booth 228

www.delcominst.com

Non-contact Sheet Resistance Meters

DENSsolutions

Booth 403

www.denssolutions.com

In Situ TEM Liquid Biasing/Heating;

In Situ TEM Gas and Heating; In Situ TEM

Heating and Biasing

Ecopia Corp.

Booth 303

www.fourpointprobes.com

Hall Effect Measurement Systems; Rapid

Thermal Processing Systems; Thermal

Evaporation and Sputtering

\section{Electron Microscopy Sciences Booth 502}

www.emsdiasum.com

Material Science Coater; Lapping and Polishing

Equipment; Wafer Cleaving Systems

\section{Gatan}

Booth 401

www.gatan.com

EM Specimen Preparation Instruments;

Direct Detection Cameras; Analytical TEM Instruments and Software

Goodfellow Corporation

Booth 627

www.goodfellowusa.com

Metals; Ceramics; Polymers
HeatWave Labs, Inc.

Booth 305

www.cathode.com

Substrate Heaters; Cathodes/Electron Guns; Ion Sources

Hitachi High Technologies

Booth 201

America, Inc.

www.hitachi-hightech.com/us

Electron Microscopes; Atomic Force

Microscopes; Thermal Analysis

Hummingbird Scientific

Booth 202

www.hummingbirdscientific.com

TEM Specimen Holders; TEM/SEM/X-Ray;

In situ TEM

ibss Group, Inc.

Booth 827

www.ibssgroup.com

In Situ Plasma Cleaner; Ex Situ Plasma Cleaner

INSTEC

Booth 730

www.instec.com

Microscopy and Spectroscopy Stages;

Electrical Probe Systems; Liquid Crystal

Measurement Systems

International Centre

Booth 302

for Diffraction Data (ICDD)

www.icdd.com

Materials Characterization Databases;

Education; Powder Diffraction Journal

IOP Publishing

www.ioppublishing.org

Journals; Magazines; ebooks

Janis Research Company, LLC Booth 301 www.janis.com

Cryogenics; Probe Stations; Cryostats

JASCO

Booth 602

www.jascoinc.com

Raman; FTIR Microscopy

JFE Shoji Electronics

Corporation

www.jfe-shoji-ele.co.jp

Zero CTE Metal; Low CTE, High TC Heat Sink Material; Minimal Fab

KLA Corporation

www.kla.com

Nanoindenters
Booth 704

Booth 231 
KP Technology USA Inc. www.kelvinprobe.com

Ambient-pressure Photoemission System; Kelvin Probe; Work Function Measurement

\section{Kurt J. Lesker Company}

Booth 200

www.lesker.com

Pure Targets and Materials; Vacuum

Components; Thin Film Deposition Systems

Lake Shore Cryotronics, Inc. Booth 327 www.lakeshore.com

Cryogenic Probe Stations; Hall Effect

Measurement Systems; Vibrating Sample

Magnetometers

Lyncée Tec SA

Booth 826

www.lynceetec.com

Digital Holography Microscope;

4D Profilometry; MEMS Analyzer

M. Braun, Inc.

Booth 605

www.mbraunusa.com

Inert Gloveboxes; Vacuum Deposition Tools;

Process Equipment

\section{Malvern Panalytical}

Booth 601

www.malvernpanalytical.com

Aeris Benchtop XRD; Zetasizer Ultra;

Empyrean

\section{MANTIS-SIGMA}

Booth 227

Www.mantis-sigma.com

UHV SPM; XPS; PVD Systems and

Components

\section{McCrone Group}

Booth 731

www.mccrone.com

Linkam Microscope Stages for Materials

Analysis; X-Ray Probe Stages; Nikon

Microscopes

\section{MilliporeSigma}

Booth 426

www.sigmaaldrich.com/materials-science

Nanomaterials; Energy and Electronics;

Biomaterials

\section{MSE Supplies LLC}

Booth 829

www.msesupplies.com

Battery Materials; Thin Film Substrates and

Targets; Processing Equipment

\section{MTI Corporation}

Booth 501

www.mtixtl.com

High Throughput XRF; Rotation Powder ALD;

Roll-to-Roll Electrospinning
National Academies of Sciences, Booth 232 Engineering, and Medicine

www.nas.edu/fellowshipsoffice

Fellowships; Research Awards; Graduate

Funding

\section{neaspec GmbH}

Booth 233

www.neaspec.com

neaSNOM; nano-FTIR; Cryo-microscopy

\section{Newport Corporation}

Booth 703

www.newport.com

Light Sources; Spectrometers

Nor-Cal Products, Inc.,

A Pfeiffer Vacuum Company

Booth 304

www.n-c.com

Custom Vacuum Chambers; Vacuum

Components; Vacuum Valves and Traps

Novocontrol America, Inc.

Booth 728

www.novocontrol.com

Dielectric Spectrometers; Impedance

Spectrometers; Electrochemistry Analyzers

NT-MDT America, Inc.

www.ntmdt.com

SPM/AFM/STM; Raman TERS; Spectroscopy

Park Systems Inc.

www.parksystems.com

Atomic Force Microscopes

\section{PicoQuant Photonics}

Booth 833

North America Inc.

www.picoquant-usa.com

Laser Sources; Photon Counting Electronics

Plasmaterials, Inc.

Booth 701

www.plasmaterials.com

Sputtering Targets; Evaporation Materials;

Backing Plates

Protochips, Inc.

Booth 726

www.protochips.com

In situ TEM Holders

Quantum Design, Inc.

Booth 727

www.qdusa.com

Magneto-Optics; Correlative Microscopy;

Lithography

\section{R.D. Mathis Company}

Booth 503

www.rdmathis.com

Evaporation Sources; Evaporation Materials;

Power Supplies; Gas Purifiers
Rigaku

Booth 205

www.rigaku.com

MiniFlex

Scienta Omicron, Inc.

Booth 204

www.scientaomicron.com

MBE; ARPES; Scanning Probe

\section{Seki Diamond Systems}

Booth 402

www.sekidiamond.com

Microwave Plasma, Hot Filament and Plasma CVD Systems

SPECS-TII, Inc.

Booth 702

Www.specs.com

XPS/UPS Equipment; Customized Systems; SPM

SPI Supplies, Booth 300

a Division of Structure Probe, Inc.

www.2spi.com

Plasma Systems; Coating Systems;

Substrates

\section{Springer Nature}

Booth 801

www.springernature.com

Books; Journals; e-Books

STAIB Instruments, Inc.

Booth 604

www.staibinstruments.com

RHEED; Auger; Surface Analysis

Ted Pella, Inc.

Booth 101

www.tedpella.com

Microscopy Supplies; Sample Preparation

Equipment and Supplies; Dimpler; Vacuum

Coaters

UC Components Inc.

Booth 729

www.uccomponents.com

Vacuum Hardware

Vigor Tech USA, LLC

Booth 733

www.vigor-glovebox.com

Gloveboxes; Purification Systems;

Clean Room Equipment

Wafer World Inc.

Booth 732

www.waferworld.com

Silicon Wafers; Germanium Wafers;

GaAs Wafers

J.A. Woollam Company, Inc. Booth 600 www.jawoollam.com

Ellipsometers; Thin Film Characterization;

Spectroscopic Ellipsometers 